\title{
COMORBIDITY CHARACTERISTICS OF ADULT PATIENTS WITH REPORTED NECK AND LOW BACK COMPLAINTS - AN OUTPATIENT CLINICAL POPULATION-BASED COHORT STUDY
}

\author{
$\underline{\text { Victor M. Pedro }}^{1,2}$ and Elena Oggero ${ }^{3,4}$ \\ ${ }^{1}$ iBrain - International Institute for the Brain, New York, NY \\ ${ }^{2}$ Director of Rhode Island Integrated Medicine, Cranston, RI \\ ${ }^{3}$ Electrical and Computer Engineering Department, University of Wyoming, Laramie, WY \\ ${ }^{4}$ Vestibular Technologies, LLC, Cheyenne, WY
}

Corresponding Author: Victor M. Pedro

International Institute for the Brain - iBRAINnyc.org - 311 East 94th St. New York, NY 10128, USA

Tel: +1 646-315-1548

Email: vpedro@iBRAINnyc.org

https://doi.org/10.34107/BiomedSciInstrum.57.04159

\begin{abstract}
In clinical practice, a comprehensive history and examination often includes questionnaires covering all systems. Physical examination is augmented by functional assessment using subjective (neck and lumbar disability indexes, NDI and LBDI respectively) and objective measures (computerized dynamic posturography, CDP). In this retrospective chart review of patients with complaints of postural instability and neck or low back pain, the presence and number of comorbidities and their classification were analyzed by age, gender, and severity of the disability. In general subjects showed higher disability in the NDI than in the LBDI (with more significant impact of the proximal vs. distal pain, joint and receptor dysfunction); they had a wide range of CDP results (the more difficult the test, the higher the number of subjects that were not able to complete it and the lower the number that had healthy balance); and on average 3.84 comorbid conditions were present, with 21 subjects presenting with 5 or more, 3 with 10 or more, and one reporting 15 comorbidities. No statistically significant differences were found for age, BMI and LBDI; sex and NDI affected metabolic comorbidities; certain tests of the CDP affected the Musculoskeletal and Other type of comorbidities. It was difficult to detect strong correlation trends that could be easily explained. Complex subjects cases complicate the possibility of doing practice based clinical research, but more importantly they create a challenge for the clinician in deciding the best course of action for treating the patient. New algorithmic assessments and integrated approaches are needed.
\end{abstract}

Keywords: Practice-based clinical research, Computerized dynamic posturography, Neck disability index, Low back disability index, Comorbidity, Autonomics

\section{INTRODUCTION}

Neurodevelopment in humankind results in an evolutionary strategic distribution of sensory receptors of various types with the greatest concentration in the rostral aspects of the neuraxis with decreasing populations of mechanoreceptors in, among others, the caudal aspects of the spinal cord and soma [1]. The importance of this distribution allows for more significant empirical contribution as well as speed and modulation of contribution into the vitals centers and human cortex for the interpretation, modulation, and execution of efferent responses not only in motor systems for survival but for more efficient execution of homeostatic functions in real-time [2]. The input of the vestibular apparatus, visual system, and mechanoreceptors, in particular, have a significant influence in not only the vital centers for survival but the ascending and descending messaging to the brain and the viscera for proper function and 